\title{
PEG-3350/Sodium Sulfate/Sodium Chloride/Potassium Chloride/Sodium Ascorbate/Ascorbic Acid-based Laxative
}

National Cancer Institute

\section{Source}

National Cancer Institute. PEG-3350/Sodium Sulfate/Sodium Chloride/Potassium

Chloride/Sodium Ascorbate/Ascorbic Acid-based Laxative. NCI Thesaurus. Code C103279.

A preparation containing the nonabsorbable polymer polyethylene glycol (PEG or macrogol) 3350 and sodium sulfate, sodium chloride, potassium chloride, sodium ascorbate and ascorbic acid with laxative activity. Upon oral administration, the PEG3350, sodium sulfate, sodium chloride, potassium chloride, sodium ascorbate and ascorbic acid-based laxative promotes the retention of water in the bowel. This increases the water content of stool, which results in increased gastrointestinal motility and evacuation of colonic contents. This results in a complete cleansing of the colon. Compared to the 4 liter PEG-3350-based preparations, this PEG-based laxative is a low volume (2 liter) preparation, which improves patients' tolerance. 